\title{
Research of Fatigue Crack Growth Based on Critical Plane Method
}

\author{
Xiao MEI ${ }^{1, \mathrm{a}}$, Shihao $\mathrm{GE}^{2, \mathrm{~b}}$ and Ying $\mathrm{ZHENG}^{3, \mathrm{c}}$ \\ ${ }^{1,2,3}$ Shanghai Maritime University, Shanghai, China \\ axiaomei@shmtu.edu.cn, ${ }^{\mathrm{b}}$ geshihao1992@163.com, ${ }^{\mathrm{c}} 18701830704 @ 163 . c 0 m$
}

\begin{abstract}
To precisely simulate the path of crack growth, a simulation of crack growth progress has been presented by using the redevelopment function of ANSYS APDL and introducing the random criterion of crack propagate directions with critical plane approach. Based on the $\mathrm{C}(\mathrm{T})$ specimen test, extract the stress fields of crack tips, and set reasonable simulation parameters to verify the simulation method. The result shows that the initiation site and propagation path of crack are basically anastomoses to the results of test, which make a guiding function for preventing the accidents of crack growth and making anti crack plan.

Keywords: fatigue crack growth; critical plane approach; finite element; APDL redevelopment
\end{abstract}

\section{Introduction}

Fatigue crack growth of metal materials has been a research focus in engineering field for a long time. It's important for guiding safety production and preventing major accidents to find crack phenomenon, study the prerequisite of initiation and unstable propagation of cracks, understand the progress of stable propagation and control the propagation of crack.

Finite element method (FEM) is one of the numerical methods that is frequently used for simulating the component crack growth, there are a lot of scholars have studied. A.O. Ayhan studied the stress intensity factors of three-dimensional comprehensive fracture and simulated with the applying of general FEM software and tetrahedral enriched element method $^{[1]}$; F. Rabold and M. Kuna realized the automated finite element simulation of fatigue crack growth in three-dimensional components with the applying of the software ProCrack, which is based on the Abaqus ${ }^{[2]}$. It's can be seen that previous researches, for example $[3,4]$, are majorly focused on the analysis and derivation of finite element theory, which are hardly applied into simulation, and recent researches tend to use cohesive element ${ }^{[5,6]}$ for simulating fractures based on the conventional finite element method, but since the influences of stress field on crackle tip are not took into consideration, cracks could only generate along the boundary of elements ${ }^{[7]}$, which means presupposed direction and phase match meshing of cracks are needed for the simulation. For this reason, different presupposed direction and meshing would lead to huge differences for the results, especially at the situation when the fracture direction is hard to be predicted ${ }^{[8]}$.

Current researches for fracture direction are majorly based on the criterion of energy release rate $(\mathrm{G} \text { criterion })^{[9,10]}$. The maximum shearing stress theory is also the commonly 
used criterion for deciding the fracture direction, but under complex loading condition, the direction of shearing stress would be hardly to decide due to the cycle load variation, and this reason has also caused the infrequent of simulation that based on the maximum shearing stress. In this paper, the fracture direction has been determined based on the maximum shearing theory, according to the stress field on crackle tips and the criterion of random fracture direction, and with the applying of redevelopment function of ANSYS APDL.

\section{Basic Theory}

Theory of Fatigue Crack Growth

Based on the theory of fracture mechanics, using periodic alternating load to a component, and the crack growth rate can be described as the Paris formula, see Eq. 1.

$$
\mathrm{da} / \mathrm{dN}=\mathrm{C}(\Delta \mathrm{K})^{\mathrm{m}} .
$$

where $\mathrm{C}, \mathrm{m}$ are material constants, $\Delta \mathrm{K}$ is the stress intensity factor amplitude, $a$ is the crack size and $N$ is the cycle number of periodic load. Crack growth has three stages of the crack initiation, subcritical crack growth and unstable fracture. When $\Delta \mathrm{K}$ is lower than threshold value $\Delta \mathrm{K}_{\mathrm{th}}$, the crack is not extend. When $\Delta \mathrm{K}>\Delta \mathrm{K}_{\mathrm{th}}$, it comes to the stage II, which is the subcritical crack growth stage, and the component fractured when it comes to the stage III . The subcritical crack growth stage has good log-linear relationship, and this stage constitutes the main part of the fatigue life under low stress periodic load. Thus, stage II is key point of the fatigue crack growth simulation.

\section{Critical Plane Determination}

The critical plane approach defines a plane(i.e. the plane with maximum shearing stress) where material fatigue failure take place, accumulating fatigue damage and estimating fatigue life in this plane, thus giving a physical explanation for fatigue damage vividly. However, principal stress orientation becomes capricious under the complex load, which makes it difficult to determine the critical plane orientation. Hence, the key point of studying fatigue problem with the critical plane approach is how to determine the critical plane accurately.

According to elasticity, a new coordinate system with common point of origin can be obtained by at most two rotations of coordinate axis in three-dimensional space; the transformation matrix can be represented as Eq. 2.

$$
\mathrm{M}=\left[\begin{array}{ccc}
\cos \theta & -\sin \theta \cos \varphi & \sin \theta \sin \varphi \\
\sin \theta & \cos \theta \cos \varphi & -\cos \theta \sin \varphi \\
0 & \sin \varphi & \cos \varphi
\end{array}\right]
$$

A new coordinate system $O-x^{\prime} y^{\prime} z$ ' is presented by rotating on $z$ axis for $\theta$ firstly and then rotating on $\mathrm{x}$ axis for $\varphi$ in the original coordinate system $O-x y z$, and the counter clockwise is positive. Define a stress tensor of the crack tip as $\sigma_{i j}(i, j=x, y, z)$, and the new stress tensor can be represented as Eq. 3

$$
\sigma^{\prime}{ }_{\mathrm{ij}}=\mathrm{M}^{\mathrm{T}} \sigma_{\mathrm{ij}} \mathrm{M} \text {. }
$$

where $\sigma_{i i}^{\prime}$ is principal stress, $\theta, \varphi$ is the azimuth of principal stress. In addition, the angle between maximum shear stress and the first principal stress was $45^{\circ}$; therefore, the direction of crack growth could be determined as long as the first principal stress of 
material is found.

\section{Strategy of Crack Growth Simulation}

The secondary development of ANSYS with APDL aims at realizing automated crack growth simulation of cracked structure under fatigue load. Redevelopment module uses Paris formula for calculating step of crack growth, and parameter C, $\mathrm{m}$ are determined by experiment. Extract the real-time stress components of crack tips by ANSYS, and combine with the critical plane approach to determine the direction of first principal stress, and the direction of maximum shear stress only has two possibilities. It is possible to determine the direction of maximum shear stress with random selection by generating a random number R.

According to the fracture mechanic, when the crack length reaches a critical crack size $a_{c}$ (decided by experiment), fast fracture would occur, that is a stopping criterion for iteration in the simulation. The strategy of crack growth simulation can be represented as Fig. 1.

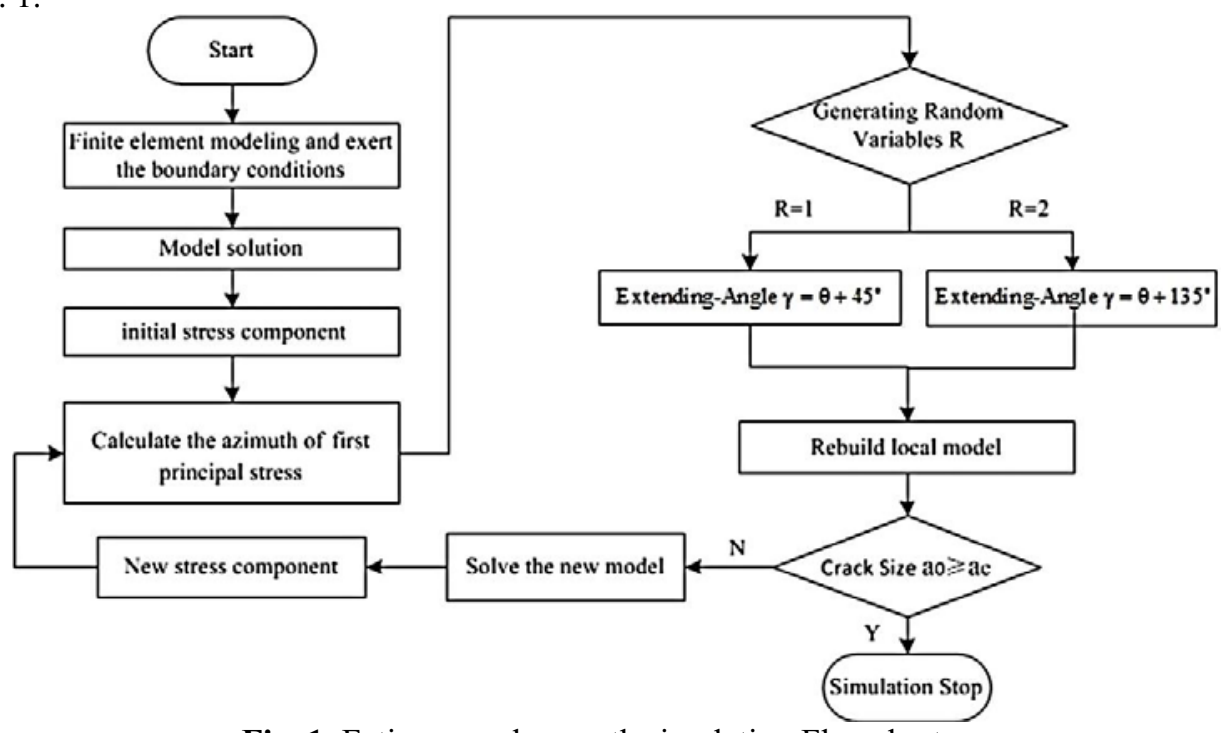

Fig. 1. Fatigue crack growth simulation Flowchart

\section{FEM Simulation and Experiment Verification}

\section{Measurement for Fatigue Crack Growth Rate}

An experiment of fatigue crack growth rate for $\mathrm{C}(\mathrm{T})$ specimen was conducted by using MTS fatigue testing machine, see Fig. 2. The material of specimen is high strength steel Q690D, tensile strength $\sigma_{b}=817.9 \mathrm{MPa}$, yield stress $\sigma_{s}=651.8 \mathrm{MPa}$, Elastic Modulus $E=206 \mathrm{GPa}$ and Poisson's ratio $v=0.3$. The standard size for specimen is shown in Fig. 3 . Define the width of specimen $W=80 \mathrm{~mm}$, and the thickness of specimen $B=8 \mathrm{~mm}$. 


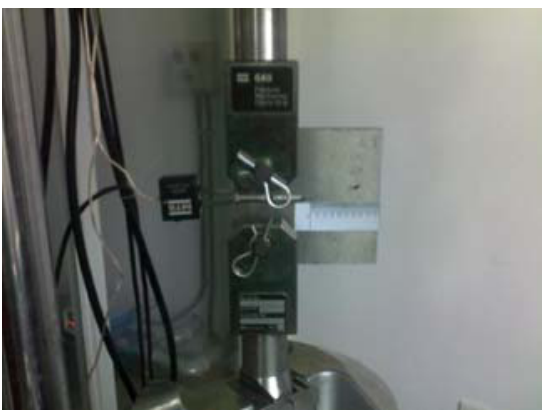

Fig. 2. Test Device
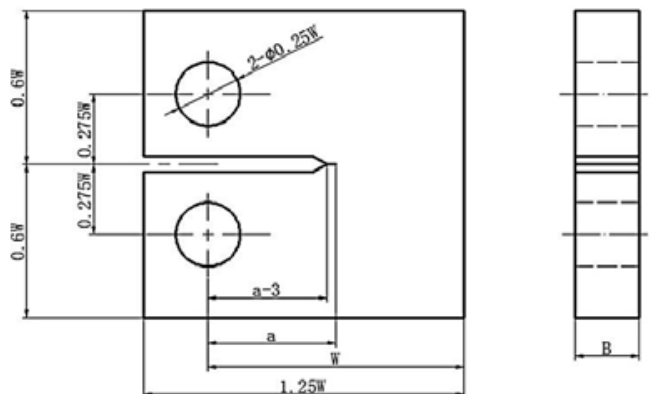

Fig. 3. Standard size for C(T) specimen

According to the test, threshold value $\Delta \mathrm{K}_{\text {th }}$ is about $0.5 \mathrm{kN} / \mathrm{mm}^{1.5}$, the experiment was conducted by using constant-amplitude loading, the value of load amplitude is $8 \mathrm{kN}$, the load ratio is 0.1 and initial stress intensity factor $\Delta \mathrm{K}=0.63 \mathrm{kN} / \mathrm{mm}^{1.5}$.

Finite Element Modeling Based on ANSYS

It is appropriate to realize the simulation of fatigue crack growth under plane stress state by using Plane183, which is a higher order 2-D, 8-node or 6-node element, and size parameters correspond with the testing specimen. Rigid region is used for simulating the displacement constraint of axis hole and defining load, see Fig. 4. Generate singular elements by using command KSCON to simulate the singularity of crack tip, see Fig. 5.

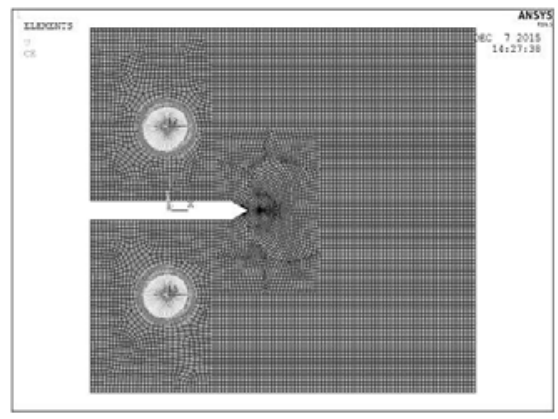

Fig. 4. Modeling with rigid region

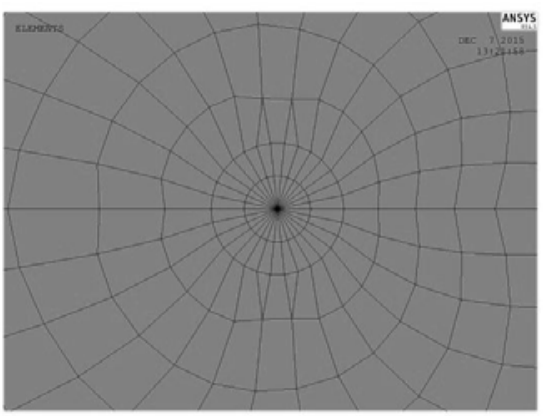

Fig.5.Grid of crack tip

\section{Results and Discussions}

After $2.4 \times 10^{5}$ times of cyclic loading during the test, the crack has propagated to 36.295 $\mathrm{mm}$, and the path of crack is shown in Figure. 8. Crack growth rate and the stress intensity factor amplitude of each cycle can be recorded by computer. Take the logarithm and fit in MATLAB, the relationship between $\mathrm{da} / \mathrm{dN}$ and $\Delta \mathrm{K}$ could be represented as Fig. 6 . 


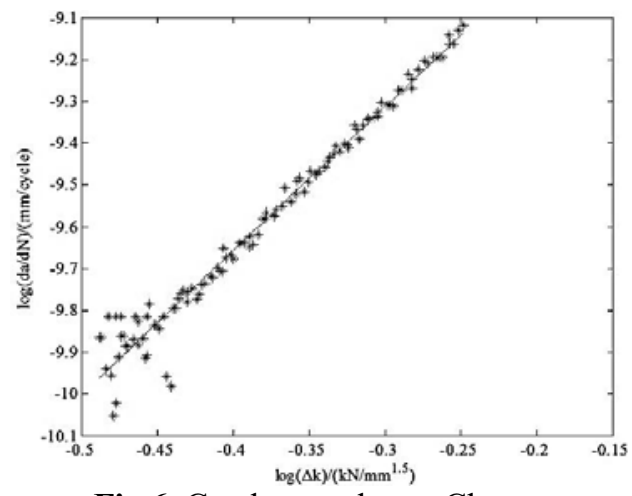

Fig.6. Crack growth rate Chart

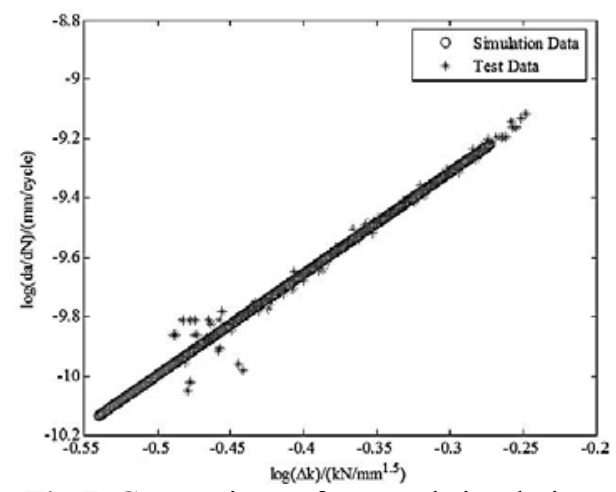

Fig.7. Comparison of test and simulation

There is good logarithm linear relationship between them, i.e. the crack is in the subcritical extension stage. And then the parameters in Paris formula could be set as $\mathrm{C}=2.53 \times 10^{-4}$ and $\mathrm{m}=3.4315$. Put these parameters into the program, the simulation of crack growth rate could be obtained, see Fig. 7. The picture shows that the simulation curve coincides with the experimental curve, which verifies the reliability of the simulation. Calculate the angle of fatigue crack growth in every cycle by the critical plane approach and combine with APDL redevelopment, the fatigue crack propagation path based on ANSYS could be obtained, see Fig. 9. Furthermore, 5 sets of parameters of crack propagation path are shown in Table 1 .

TABLE 1 PARAMETERS OF FATIGUE CRACK GROWTH SIMULATION

\begin{tabular}{cccccc}
\hline Step & 1 & 2 & 3 & 4 & 5 \\
Extending-Angle $\gamma$ & $45^{\circ}$ & $-64.8^{\circ}$ & $-12.2^{\circ}$ & $52.6^{\circ}$ & $19.8^{\circ}$ \\
First principal & 573.002 & 601.428 & 549.859 & 673.141 & 572.627 \\
stress[Mpa] & & & & & \\
\hline
\end{tabular}

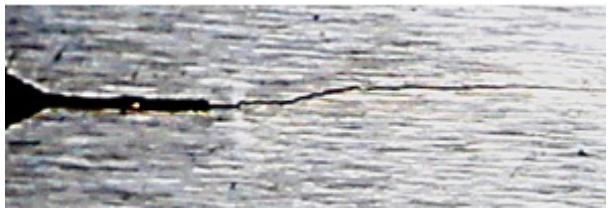

Fig. 8. Crack path of experiment

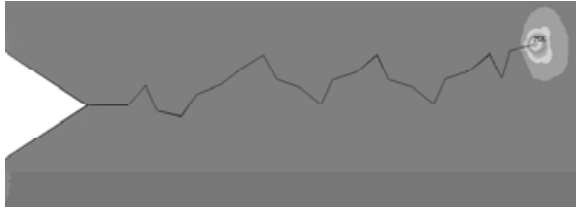

Fig. 9. Crack path of simulation

In Fig. 9, the crack moves forward in serrated shape, and this result is conformed to the test result that there is a $45^{\circ}$ angle between the direction of micro crack propagation and the stress axis, and the macro crack propagates vertically to the direction of stress axis. For this sake, the correctness of ANSYS simulation and the applicability of the critical plane approach could be verified.

\section{Conclusion}

1. Realize a strategy of dynamic re-meshing and model rebuilding based on APDL redevelopment.

2. To reduce human interference, a method that extract the stress fields of crack tips, combine with the critical plane approach and the criterion of random fracture direction 
without presupposed direction has been presented.

3. Analyze a classical example and compare with the experiment that verifies the reliability and the feasibility of the simulation of fatigue crack growth based on ANSYS redevelopment.

\section{References}

1. A.O. Ayhan. Three-dimensional fracture analysis using tetrahedral enriched elements and fully unstructured mesh[J]. International Journal of Solids and Structures, 2011, 48(3/4):492-505.

2. F. Rabold, M. Kuna. Automated Finite Element Simulation of Fatigue Crack Growth in Three-dimensional Structures with the Software System ProCrack[J]. Procedia Materials Science, 2014, 3:1099-1104.

3. Yang Qingsheng, Yang Wei. Finite element simulation of fracture process $[\mathrm{J}]$. Chinese Journal of Computational Mechanics, 1997, 04:33-38.

4. Jia Jianjun, Peng Yinghong, Ruan Xueyu. A grid technology of crack propagation simulation based on FEM[J]. Die and Mould Technology, 2001, 05:4-6.

5. R. Moslemian, A.M. Karlsson, C. Berggreen. Accelerated fatigue crack growth simulation in a bimaterial interface[J]. International Journal of Fatigue, 2011, 3312:1526-1532.

6. P. Beaurepaire, G.I. Schuëller. Modeling of the variability of fatigue crack growth using cohesive zone elements[J]. Engineering Fracture Mechanics, 2011, 7812:2399-2413.

7. Su Shaopu, Dong Dengke, Zhang Haiying. Fatigue Crack Growth Analysis Based on Abaqus /Python [J]. Science Technology and Engineering, 2015, 27:193-198.

8. Chen Guohua, Chen Lingjie. Solution-dependent Path Crack Propagation Simulation and Its Application[J]. Pressure Vessel Technology, 2012, 10:36-40.

9. B.R. Davis, P.A. Wawrzynek, C.G. Hwang, A.R. Ingraffea. Decomposition of 3-D mixed-mode energy release rates using the virtual crack extension method[J]. Engineering Fracture Mechanics, 2014,:382-405.

10. Fan Chengye, P.-Y. Ben Jar, J.J. Roger Cheng. Prediction of energy release rates for crack growth using FEM-based energy derivative technique[J]. Engineering Fracture Mechanics, 2006, 748:1243-1254. 